\title{
ITRF Densification and Continuous Realization by the IGS
}

\author{
G. Blewitt ${ }^{\dagger}$, C. Boucher, P. B. H. Davies, M. B. Heflin, T. A. Herring and J. Kouba \\ ${ }^{\dagger}$ Department of Geomatics, University of Newcastle upon Tyne \\ Newcastle upon Tyne, NE1 7RU, United Kingdom
}

\begin{abstract}
The tools and infrastructure are now in place to realize a global, terrestrial, kinematic reference frame with few millimeter precision, which is both spatially dense, and can be reliably updated on a frequent basis (e.g., monthly). A procedure is outlined by which IERS can continuously realize the ITRF using methodology developed by the International GPS Service (IGS). However, spatial densification not only requires such a processing scheme, but also requires the recruitment of interested groups to participate in this venture.
\end{abstract}

\section{INTRODUCTION}

It is appropriate that Geodesy, a discipline over 2000 years old, is approaching a watershed as we move into the new millenium. Around 200 B.C., Eratosthenes used the difference in direction of the sun's rays between Syene and Alexandria to infer the radius of the Earth, producing an answer only 2\% in error [Torge, 1980]. (Careful as he was, this surely required a degree of luck). Within the last two decades, the development of space geodesy has allowed us to measure the $12700 \mathrm{~km}$ distance across the Earth between any two points with an accuracy of $1 \mathrm{~cm}$, which is $0.0000001 \%$, or one part per billion. This requires no luck, in the sense that the results are repeatable at this level; the various techniques ( SLR, VLBI, GPS, and DORIS) also agree at this level.

The real breakthrough in this last decade of the millenium, is that positioning with 1-cm accuracy can now be routinely achieved on a daily basis, by anyone armed with a single dual-frequency GPS receiver, appropriate software, and products from the International GPS Service for Geodynamics (IGS). With this revolution in the making, it is right for us to step back and reconsider our, perhaps, outdated notions of reference frames. The idea of control networks has evolved with space geodesy, but the basic idea has until recently 
remained as Bomford [1980] describes triangulation control networks: "To constitute the main framework on which less precise observations may be based...”

Indeed, this describes the "fiducial concept", the idea prevalent in the 1980's that precise VLBI or SLR measurements could provide the framework within which densification could be achieved using GPS. Although GPS has since become a truely global technique, similar ideas are also reflected in the IGS program to densify the IERS Terrestrial Reference Frame (ITRF). This program is again based on the principles of heirarchical networks, starting with the IGS Global Network, which is then densified by regional networks to produce the IGS Polyhedron (100-200 stations) [Blewitt et al., 1993a, 1995].

This IGS Polyhedron can then act as "active" control points, relative to which users can position themselves. However, it is a misconception, perhaps too commonly held, to think that the reasons for the Polyhedron are analogous to the triangulation network heirarchy described by Bomford. GPS relative positioning is now essentially independent of distance, using the right tools and techniques, so why do we need the Polyhedron?

The real issue is one of reference frame, and the fact that all stations are moving at a level which is very significant compared to the positioning precision. We need the Polyhedron as an active reference frame, so that we can better interpret what our positional coordinates actually mean. As a concrete example, major earthquakes can displace these so-called "stable reference points" on a regional scale [Blewitt et al., 1993b, Bock et al., 1993]. In this example, we must be careful to define the time evolution of the frame such that points well outside the area of co-seismic deformation do not appear to be displaced due to the earthquake. This sounds so obvious, yet it is no longer obvious what to do when unpredictable motions are not strictly regional phenomena, but extend over all scales. This fact has been realized ever since the network design stage of IGS:

"We do not enjoy the benefit of potential fields or elastic waves to define a physical averaging of pointwise properties of the Earth, so we must rely on a hierarchy of networks, with sparser regional networks serving as fiducial sites in the survey of denser local networks" [Minster et al., p.24, 1993]

We can therefore view the Polyhedron as providing a primary kinematic description of the Earth's shape as it changes. Although the "reference system" can account for predictable changes as prescribed by conventional models, only the "reference frame" can account for true motions. We should therefore emphasize the kinematic aspects of reference frames if we are to keep pace with these technological advances. How do develop and realize this is the purpose of this paper.

\section{IGS DENSIFICATION PROGRAM}

After several years of planning [Mueller and Beutler, 1992], the International GPS Service for Geodynamics (IGS) was officially established in 1993 by the International Association of Geodesy. Ever since an initial pilot phase beginning June 1992, the IGS has been coordinating the operations and analysis a global network of GPS stations. The IGS officially commenced operations in January 1994, by which time approximately 40 to 50 IGS stations had become operational.

The expanding global network of high precision GPS receivers (Figure 1) was seen to present an opportunity to produce a reference frame which is (i) dense, (ii) of a reasonably homegeneous quality, (iii) of few-millimeter accuracy on a global scale, (iv) readily 
accessible to GPS users, and (v) ideal for monitoring variations in the Earth's shape, and for providing kinematic boundary conditions for regional and local geodetic studies [Blewitt et al. 1993a, 1995]. The challenge was to be able to analyze cohesively the data from an ever increasing number of receivers, such that near-optimal solutions could be produced. Although ideally all data should be analyzed simultaneously to produce a single solution, in practise this is computationally prohibitive

This led to the "distributed processing approach," which, at the algorithm level, partitions the problem into manageable segments [Figure 1], and, at the organizational level, delegates responsibility to analysis centers who would naturally have an interest in the quality of the solutions. Another characteristic of this approach is a level of redundancy, such that a meaningful quality assessment can be made by other, independent groups. Distributed processing was developed as a method which could be carried out as a natural extension to the existing operations of the IGS.

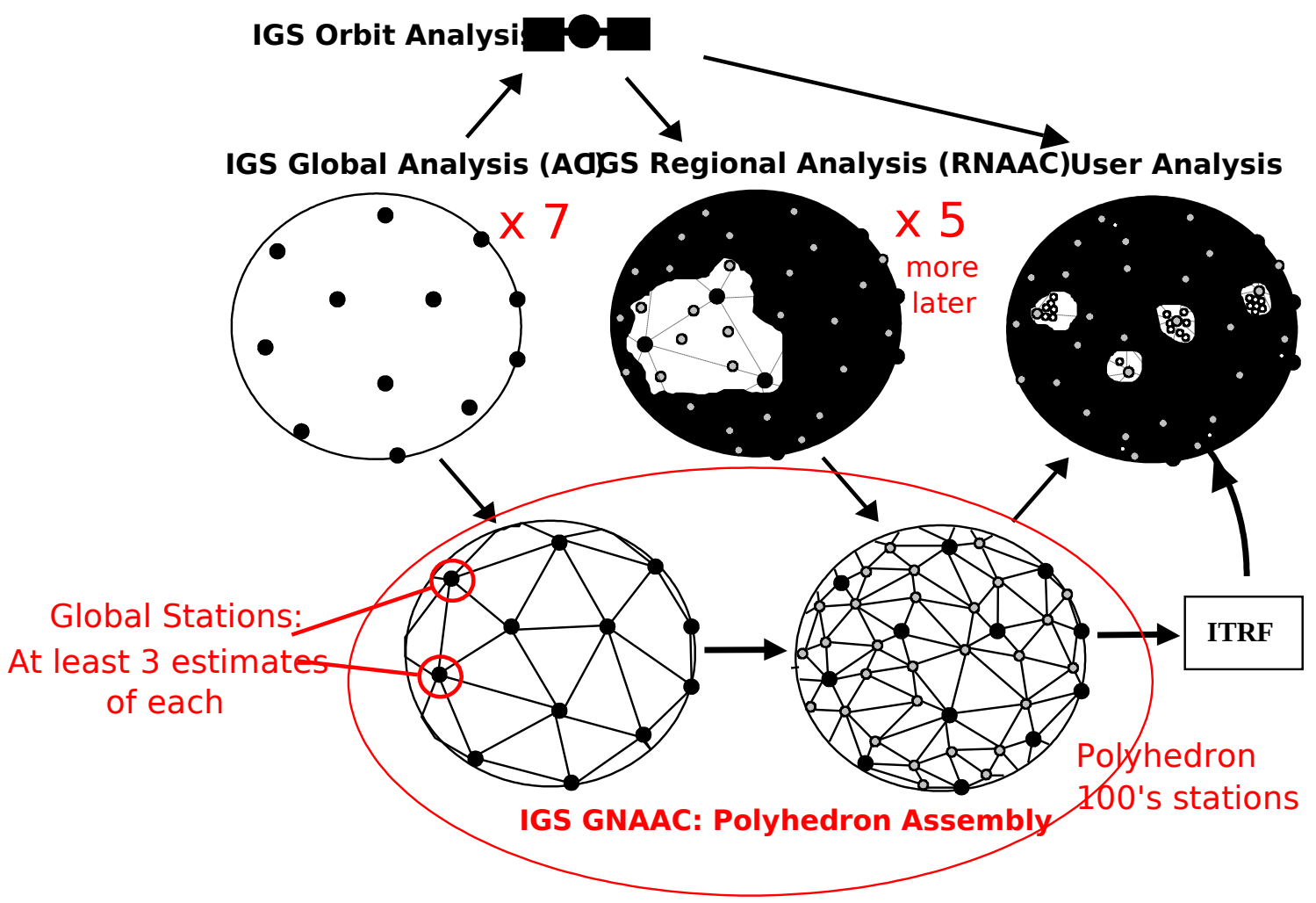

Figure 1: Schematic explanation of the distributed processing approach. Our proposal is for science groups to operate as RNAACs. The GNAACs would then take care of reference frame consistency, and input into ITRF.

Following a planning workshop at JPL in December 1994 [IGS, 1995], a pilot program was initiated in September 1995 to test these ideas. Global Network Associate Analysis Centers (GNAACs) were set up at Newcastle University, MIT, and JPL. A format was developed for the exchange of coordinate solutions, covariance matrices, and site information (SINEX format) [SINEX Working Group, 1996]. Initially these GNAAC's 
combined solutions for global network station coordinates provided every week by the seven Analysis Centers, producing a single unified SINEX file. Approximately one year later, Regional Network Analysis Centers (RNAACs) began submitting regional GPS solutions, computed using weekly published IGS orbit solutions. These regional solutions were then assimilated into the unified global solution by the GNAACs, what is known as the "IGS polyhedron solution."

Although currently undergoing final review, the pilot program has been viewed broadly as a success, demonstrating few-millimeter repeatability in weekly solutions for geocentric coordinates of not only the global stations, but also the regional stations. However the actual process of densification (new GPS stations) is still less than adequate in many parts of the globe. For example, tide-gauge benchmark monitoring could help. Additional GPS stations installed at island tide-gauge sites will undoubtedly be greatly welcomed by IGS, especially as oceanic regions of the globe are systematically undersampled (which is the primary reason for the lack of stations in the ocean-rich southern hemisphere). Furthermore, the IGS Densification Program provides a natural way for science groups to participate in IGS. We expand on this point in a later section.

\section{IGS Global Network Associate Analysis Centers: Methods and Results}

Blewitt et al. [1995] discuss the following components of the GNAAC activities (previously called “Type Two Analysis” during the planning stages): (i) detection of interagency information discrepancies (e.g. in antenna heights); (ii) monitoring of solution consistencies (inter-agency, and with respect to ITRF); (iii) weekly publication of a combined global solution; (iv) weekly publication of an IGS polyhedron solution (global plus regional networks); (v) periodic publication of kinematic solutions (e.g., station height velocity, plate tectonic Euler vectors, etc.), with submission to the International Earth Rotation Service (IERS) with the goal of improving the ITRF.

Global Network Associate Analysis Centers (GNAACs), at Newcastle University (NCL), MIT, and JPL have so far been operating during the pilot phase of the IGS Densification Program. All three GNAACs have in principal been using similar (but not identical) approches and that results are quite comparable. For the purpose of this paper, methods and results from NCL are briefly highlighted

Now two years since the inception of the IGS Densification Pilot Program, the NCL GNAAC is continuously achieving all stated objectives [Davies and Blewitt, 1996, 1997]. Taking the most recent submission at the time of writing, coordinate solutions for 132 stations are presented, of which approximately 50\% are global stations (defined as being analyzed by at least 3 Analysis Centers), and 50\% are regional. A total of 54 regional station solutions derive from 3 RNAACs which cover South America, Europe, and Japan.

We have developed combination procedures [Davies and Blewitt, 1996, 1997] which aim to (1) minimize bias from datum assumptions, (2) minimise bias from unrealistic covariance matrices; (3) utilize the inherent redundancy of overlapping networks to remove outliers objectively. The first is achieved by applying a loosening transformation to each input covariance matrix [Blewitt, 1997], which can be interpreted as the inverse of reference frame projection [Blewitt, 1992]. The second is achieved by variance component estimation [Rao and Kleffe, 1988; Sahin et al. 1992]. The third is achieved by applying reliability analysis theory [Kosters and Kok 1989]. 
Our weekly, long-term repeatability in station height has a best case value of $3 \mathrm{~mm}$, median of $7 \mathrm{~mm}$, and worst case of $19 \mathrm{~mm}$. This is to be compared with the best Analysis Center solutions (best case $4 \mathrm{~mm}$, median $9 \mathrm{~mm}$, worst case $>30 \mathrm{~mm}$ ). We conclude that GNAAC analysis not only provides a consistent unique solution, but also a more reliable solution (in the statistical sense of the word). The IGS Densification Program methodology should not be viewed as compromising solution quality, but rather as a preferred alternative to unilateral analysis.

In summary, GNAAC results show that the combined solutions produce coordinate time series with a smaller variance than any individual AC network solution, even more so for stations with the worst levels of precision. This indicates that reliable outlier detection, due to redundancy from the multiple solutions, is a major reason for this improvement. Therefore, we can conclude that GNAAC solutions are not only more precise, but more reliable than any individual contributing AC solution.

\section{IERS STRATEGY FOR ITRF}

Since its establishment in 1988, the International Earth Rotation Service (IERS) published on an annual basis a new realization of the International Terrestrial Reference System (ITRS). Each year, the IERS Central Bureau collected among contributing analysis centers their solutions for Earth Rotation Parameters together with the associated Terrestrial Reference Frame (TRF) (and Celestial if available) data. These contributions are currently available for several space techniques: SLR, LLR, VLBI, GPS and DORIS.

Such a realization is now widely known under the label ITRFyy. Up to $y y=94$, this solution was obtained by a combination of all data submitted to the IERS Central Bureau at the begining of $y y+1$. It was assumed that all individual analysis centers would provide by this was their best and most complete individual solution, in particular including data for the year yy.

The succession of these results, from ITRF88 to ITRF94, did actually show an improvement both in accuracy for positions of stations as well as in the geographical coverage of the corresponding network. Nevertheless, the accuracy reached was ranging from a few millimeters to a few centimeters, and a lot of details concerning modeling were raised at this level (in particular relativistic and geodynamical effects).

At that time, IERS decided to establish a primary solution upon specifications of an international working group (WG on ITRF datum). Although this is exactly a task for this WG to recommend the precise definition of what should be a primary ITRF solution, we can agree that the main concept is to provide a consistent, homogeneous set of positions (and time variations) at the subcentimetric level (in precision and reliability) for a well distributed network. This work is presently in progress, in particular by performing research (experimental and theoretical) on the geocenter, which is one of the new topics to be investigated before establishing these general recommendations.

Considering on the other hand the numerous and increasing requirements of the users, mostly outside the restricted IERS community, IERS decided to continue the annual series of ITRF publications for a complete solution along the line of the series up to ITRF94.

Starting for $y y=96$, ITRFyy is now specified to be the best complete solution produced on an annual basis by IERS. In particular, any data set expected to bring a useful contribution to this goal should be included, and not only the data from the annual 
submission to the Central Bureau. Specific work is currently underway for GPS and DORIS contributions, with the possibility of producing updated realizations of ITRFyy, perhaps every month. Both techniques are growing significantly and providing each year new stations and improved present day position quality.

\section{Cooperation with IGS}

Close cooperation with IGS is very useful and already under expansion, with mutual benefits:

- since the begining, the IGS organization stimulated individual analysis centers to submit solutions in response to the IERS Central Bureau's annual call for data.

- IGS uses for operational purposes ITRFyy solutions. IGS represents a strong user group in favor of these annual solutions. ITRF96 will therefore be very welcome.

- conversely, the GPS contribution for ITRF96 is very strong, including results from IGS densification pilot experiment.

- using these ITRF solutions (or maybe in the future a cleaner primary solution which is under consideration by a ad hoc working group on ITRF datum), IGS could consider by its ITRF densification strategy to provide an operational TRF solution similar to rapid service for EOP.

We propose that routine monthly GNAAC solutions be submitted to the IERS as input to a continuous realization of the ITRF. Not only would this have benefits from the standpoint of reliability, but also non-linear station motion would also be represented by such an approach (e.g., due to possible monument instability, the earthquake cycle, or seasonal loading effects). This approach would also be particulary useful for recently installed stations whose velocities are not yet very well determined.

As an IERS user group, IGS could then use the continuously updated realization of ITRF for its products. Of course, users such as IGS may not wish to update its reference frame every month, however the opportunity will always be there to perform a frame update when needed. For example, IGS recently experienced a slight degradation in the network of available primary stations used to define the frame of the orbits and Earth rotation parameters. Although IGS considered producing its own realization of ITRF to improve the situation, it was decided to wait for the imminent ITRF96. Clearly, a set of sub-annual reference frame realizations would have been useful under these circumstances.

\section{ORGANIZATIONAL STRUCTURE FOR ITRF DENSIFICATION}

We can broaden the definition of "ITRF Densification" to mean temporal as well as spatial densification. For temporal densification, we have already described a scheme whereby IGS can contribute to, say, monthly realizations of the ITRF. Of course, other space geodetic techniques (e.g., DORIS).could also be coordinated towards this goal.

For spatial densification, we have described a scheme designed on the principle of distributed processing, which easily allows the incorporation of data from many permanent GPS networks. We haven't explored how RNAAC's can be “recruited" to ensure a good distribution of stations to suit a variety of scientific purposes. Since IGS is 
a service organization, and not primarily in the business of scientific investigation, we should promote the idea that RNAAC's be connected to some science group, which has its own objectives and agenda. This should ensure that scientific needs are met, while decentralizing the operational burden away from other IGS components.

An organizational structure for this is presented in Figure 2, which reproduces a diagram presented at a recent workshop for monitoring sea level held at JPL in March 1997 [Blewitt et al., 1997]. Simple lines connecting the boxes indicate the organizational hierarchy. Arrows indicate data flow.

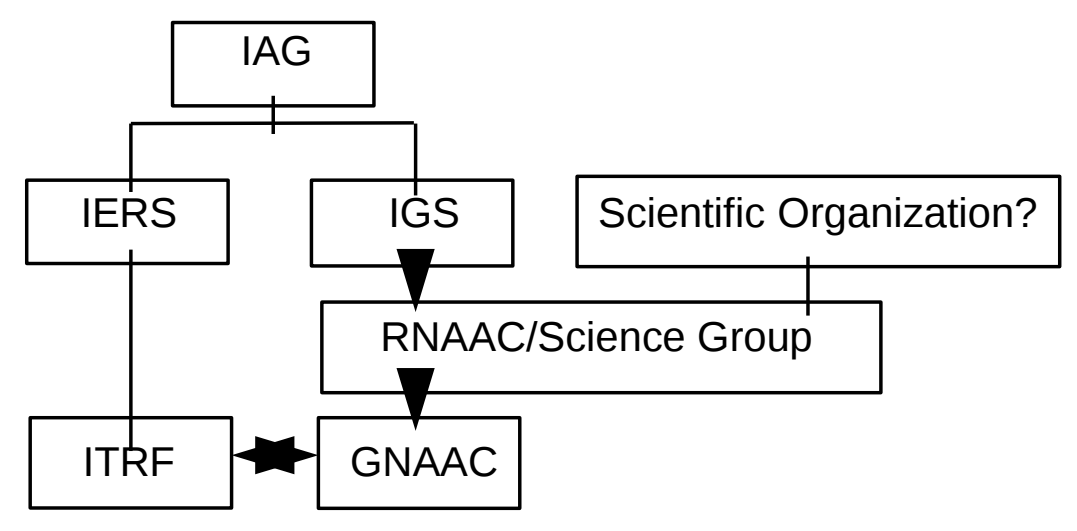

Figure 2: Chart illustrating organizational links and data flow to facilitate the activity of tide-gauge benchmark monitoring (explained in text).

Figure 3 expands this idea specifically for the community interested in monitoring tide gauge benchmarks. We use this here as an example of how Figure 2 might be practically realized . Starting with the bottom right hand side, we have the goal of this organization, which is the production of a database (DB) of the coordinates and velocities of tide gauge benchmarks available at the Permanent Service for Mean Sea Level (PSMSL), which formally reports to the Commission of Mean Sea Level and Tides (CMSLT), under the umbrella of the International Association for the Physical Sciences of the Oceans (IAPSO).

The structure described so far is essentially in place. What remains to be done, is to include GPS data from tide gauge sites into the dataflow, which would be analyzed by new RNAAC's. Figure 3 shows each RNAAC as a part of a science group which falls under the International Association of Geodesy (IAG) through the Special Commission 8 on Sea Level and Ice Sheet Variations (SC8). Special Commission 8's terms of reference look as if they have been written especially for this task, since they not only mention geodetic observing programs to investigate sea level change, but also interdisciplinary communication betwee geodesists, geophysicists, and oceanographers. Science groups are also connected to the CMSLT to make the collaboration with oceanographers explicit, and for the practical necessity for expertise on tide gauge selection. It would be natural for science groups to be regional, given that they act as RNAACs. To complete the loop, the Science Groups access both the tide gauge records and the geodetic records from the PSMSL for scientific interpretation. 


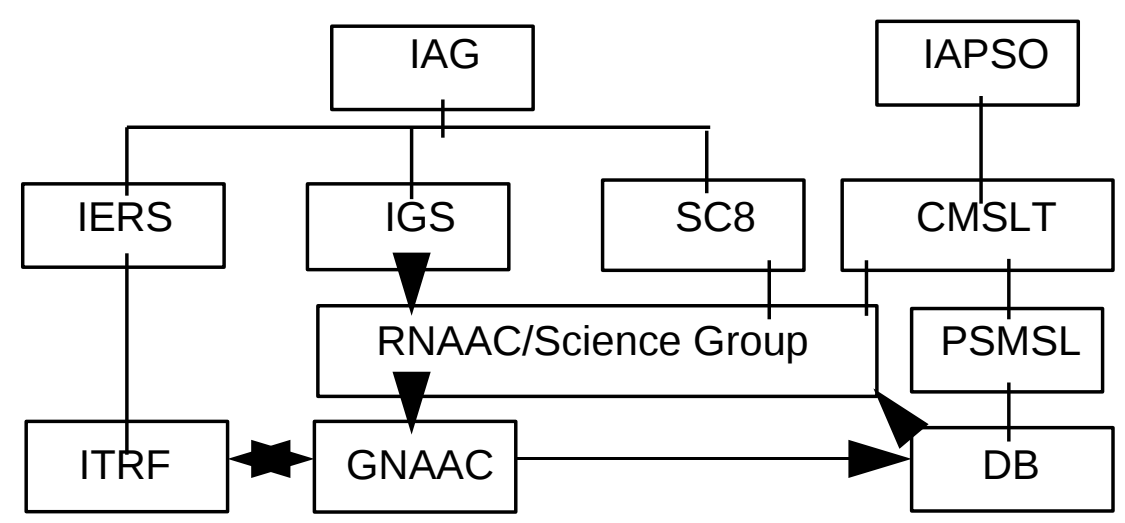

Figure 3: Chart illustrating organizational links and data flow to facilitate the activity of tide-gauge benchmark monitoring.

\section{CONCLUSIONS}

A procedure has been outlined by which IERS can continuously realize the ITRF, using IGS methodology which has been tested over the past 2 years during its ITRF Densification Pilot Project. We propose that this project move out of the pilot phase, and be made official. This will involve the resolution of some technical details and coordination between IGS and IERS, however much of those details have been already resolved with the development of the SINEX format for the exchange of geodetic solutions.

We have also pointed out that spatial densification not only requires a processing scheme, but also requires the recruitment of interested groups to operate the networks and participate in the scheme. We have identified scientific groups as likely candidates for operating IGS Regional Network Associate Analysis Centers. We show, for example, that the IGS, IERS, and the tide gauge benchmark community can be served by RNAAC's serving as part of the IAG Special Commission 8 "Sea Level and Ice Sheets", in collaboration with the IAPSO Commission for Mean Sea Level and Tides.

In conclusion, although there is certainly room for improvement in geodetic techniques and reference frame definition, ultimately the user relies on products which may not necessarily reflect the best currently achievable accuracy. What we have presented here is one way forward to improve the ITRF as a useful, accurate, and reliable product.

\section{REFERENCES}

Blewitt, G, "GPS Data Processing Methodology: From Theory to Applications," in GPS Geodesy, Eds. A. Kleusberg and P.J.G. Teunissen, Lecture Notes on Earth Sciences, Springer (in press, 1997) 
Blewitt, G., P.B.H. Davies, T.Gregorius, R.S. Kawar, and D.U. Sanli, Sustainable geodetic monitoring of the natural environment using the IGS, in Methods for Monitoring Sea Level, GPS and Tide Gauge Benchmark Monitoring, GPS Altimeter Calibration, Jet Propulsion Laboratory, Pasadena, Calif. USA (in press 1997)

Blewitt, G., Y. Bock, and J. Kouba, "Constructing the IGS polyhedron by distributed processing," in Proc. of the IGS Workshop, ed. by J. Zumberge, IGS Central Bureau, Pasadena, Calif., USA, p. 21-36 (1995)

Blewitt, G., Y. Bock, and G. Gendt, "Regional clusters and distributed processing," in Proc. of the IGS Analysis Center Workshop, Ed. by J. Kouba, pp. 62-91, Ottawa, Canada (1993a).

Blewitt, G., M.B. Heflin, K.J. Hurst, D.C. Jefferson, F.H. Webb, and J.F. Zumberge, "Absolute far-field displacements from the 28 June 1992 Landers earthquake sequence," Nature, Vol. 361, p. 340-342 (1993b)

Blewitt, G., M.B. Heflin, F.H. Webb, U.J. Lindqwister, and R. P. Malla, "Global coordinates with centimeter accuracy in the International Terrestrial Reference Frame using the Global Positioning System," Geophysical Research Letters, 19, pp. 853-856 (1992).

Bock. Y., Agnew, D.C., Fang, P., J.F. Genrich, B.H. Hager, T.A. Herring, K.W. Hudnut, R.W. King, S. Larsen, J-B. Minster, K. Stark, S. Wdowinski and Wyatt, F.K., "Detection of crustal deformation from the Landers earthquake sequence using continuous geodetic measurements,” Nature, 361, 337-340 (1993)

Bomford, G., Geodesy, Clarendon Press, Oxford (1980)

Davies, P.B.H. and G. Blewitt, "Newcastle upon Tyne Global Network Associate Analysis Centre Annual Report 1996,” IGS Annual Report 1996, p. 237-252, IGS Central Bureau, Jet Propulsion Lab., Pasadena, California (1997)

Davies P.B.H. and G. Blewitt, "Newcastle upon Tyne IGS Global Network Associate Analysis Centre Annual Report 1995,” IGS Annual Report 1995, p. 189-202, IGS Central Bureau, Jet Propulsion Lab., Pasadena, California (1996)

IGS, Densification of the IERS Terrestrial Reference Frame through Regional GPS Networks, Eds. J.F. Zumberge and R. Liu, IGS Central Bureau, Jet Propulsion Laboratory, Pasadena, California (1995)

Kosters, A.J.M. and J.J. Kok, "Statistical testing and quality analyusis of aobservations and transformation parameters in combining 3-dimensional networks," Geodetic Computing Centre (LGR), Faculty of Geodesy, Delft Univ. of Tech., presented at Symp. S102, IAG Congress, 3-12, Aug. 1989, Edinburgh (1989)

Minster J.B., M. Bevis, Y. Bock, C. Boucher, O. Columbo, B. Engen, A.M. Finkelstein, H. Frey, B. Hager, T. Kato, S. Lichten, P. Morgan, W. Prescott, C. Reigber, S. Rekkedal, B. Schutz, H. Tsuji, and V. Velikhov, "Network design considerations for the International GPS Geodynamics Service," p.23-32, in IAG Symposia 109: Permanent Satellite Tracking Networks for Geodesy and Geodynamics, Ed. G. L. Mader, Springer Verlag, Berlin (1993)

Mueller, I. and G. Beutler, "The International GPS Service for Geodynamics Development and current structure," in Proc. of the 6th Int. Geodetic Symp. on Satellite Positioning, Columbus, Ohio, pp. 823-835, 1992 
SINEX Working Group, “SINEX - Solution Independent EXchange format,” Appendix 1 in IGS 1996 Analysis Centre Workshop Proceedings, Eds. R.E. Neilan, P.A. Van Scoy, and J.F. Zumberge, pp. 223-276, IGS Central Bureau, Jet Propulsion Laboratory, Pasadena, California (1996)

Rao C.R. and J. Kleffe, Estimation of Variance Components and Applications, Elsevier, Amsterdam (1988)

Sahin, M., P.A. Cross, and P.C. Sellers, "Variance component estimation applied to satellite laser ranging,” Bulletin Geodesique, 66, 284 (1992)

Torge, W., Geodesy, Walter de Gruyter \& Co., Berlin (1980) 